\title{
Functional Relation Between Signal Distortion and a Figure of Merit for Nonlinear Process in Dispersion-managed Optical Transmission
}

\author{
Sungman Kim* \\ Department of Electronic Engineering, Kyungsung University, \\ Daeyeon-3-dong, Nam-gu, Busan 608-736, Korea
}

(Received March 26, 2012 : revised April 27, 2012 : accepted May 7, 2012)

\begin{abstract}
We show that the minimum EOP (eye-opening penalty) obtained by tunable dispersion compensation is a function of a figure of merit for a nonlinear process, $\mathrm{I}_{0} \mathrm{~L}_{\text {eff, }}$, where $\mathrm{I}_{0}$ is the optical intensity and $\mathrm{L}_{\text {eff }}$ is the effective length of the interaction region. Using this rule, we do not need to conduct nonlinear simulations in all the cases of signal power and transmission length to obtain the signal distortion in dispersion-managed optical transmission. Instead, we need to conduct a simulation in only one case of a signal power and find the functional relation, and then we can obtain the values of the signal distortion in other cases using the discovered functional relation. This technique can reduce the number of nonlinear simulations to less than $10 \%$.
\end{abstract}

Keywords: Nonlinear optical fiber transmission, Eye-opening penalty, Signal distortion, Nonlinear simulation, Dispersion-managed optical transmission

OCIS codes : (060.2330) Fiber optics communications; (060.2360) Fiber optics links and subsystems; (060.4370) Nonlinear optics, Fibers

\section{INTRODUCTION}

Nonlinear effects in optical fibers such as self-phase modulation (SPM) have been considered as a major impairment in the dispersion-managed long-haul optical fiber transmission systems. There has been much work in attempting to understand the nonlinear effects as a function of system parameters such as optical power per channel, transmission distance, and chromatic dispersion [1-5]. In particular, there have been a lot of efforts to find the optimal dispersion-compensation ratio in nonlinear optical transmission [6-9].

To investigate the nonlinear effects as a function of the system parameters, we usually conduct numerical simulations because it is not easy to change all the system parameters freely in a long-haul transmission experiment. However, nonlinear simulation also takes a lot of time if we want to conduct for all the possible cases. Therefore, if we find a way to reduce the nonlinear simulation cases, it will be very helpful for doing research on nonlinear effects.

In this paper, we report that the signal distortion in dispersion-managed optical transmission systems can be expressed by using a figure of merit for the efficiency of a nonlinear process [10], $\mathrm{I}_{0} \mathrm{~L}_{\text {eff, }}$, where $\mathrm{I}_{0}$ is the optical intensity and $\mathrm{L}_{\text {eff }}$ is the effective length of the interaction region. In other words, the signal distortion at the optimal dispersion compensation is only a function of $\mathrm{I}_{0} \mathrm{~L}_{\text {eff }}$ although the optical power per channel or transmission length is changed. Even though we have found this phenomenon from nonlinear simulation results, we will also show an analytical derivation that the signal distortion at the optimal dispersion compensation is a function of $\mathrm{I}_{0} \mathrm{~L}_{\text {eff }}$ in the case of a Gaussian pulse.

\section{SIGNAL DISTORTION IN DISPERSION-MANAGED TRANSMISSION}

It is well known that signal distortion is minimized when dispersion of the transmission fiber is exactly compensated in the linear optical transmission. However, in the nonlinear transmission case, the optimal value of dispersion compensation is changed due to the effect of SPM [11]. Fig. 1 shows an example of eye-opening penalty (EOP), a measure

\footnotetext{
*Corresponding author: sungman@ks.ac.kr

Color versions of one or more of the figures in this paper are available online.
} 


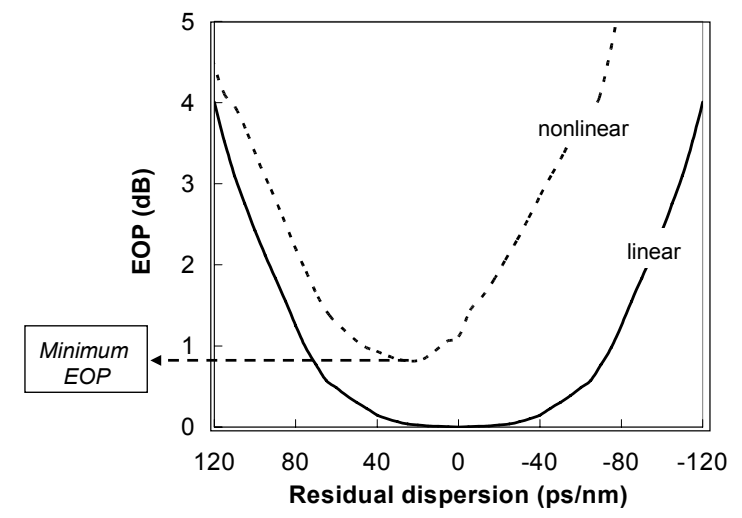

FIG. 1. Eye-opening penalty (EOP) due to dispersion and $\mathrm{SPM}$ in $40-\mathrm{Gb} / \mathrm{s}$ transmission over $720 \mathrm{~km}$ of nonzero dispersion-shifted fiber (NZDSF, D $=4 \mathrm{ps} / \mathrm{nm} / \mathrm{km}$ ) with a launch power of $-4 \mathrm{dBm}$. Solid line is the simulation result without considering the nonlinear effect and dotted line is with considering the nonlinear effect. The definition of the minimum EOP is also illustrated.

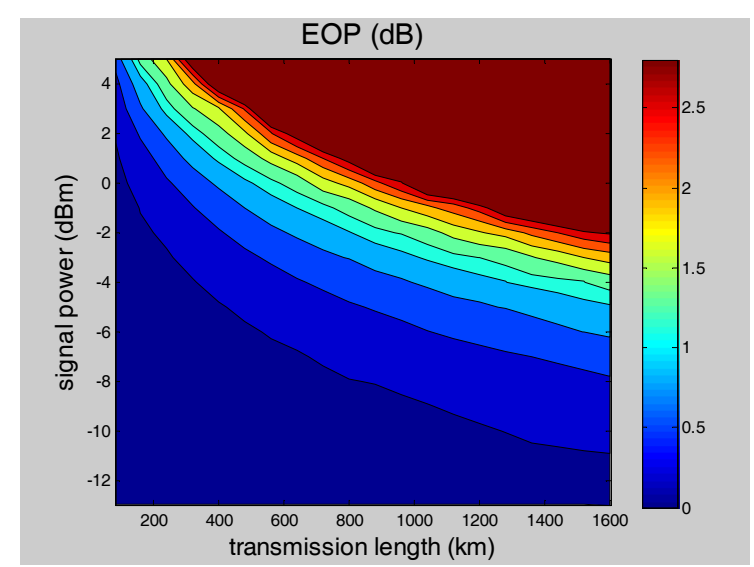

FIG. 2. The minimum EOP with the optimal dispersion compensation as a function of signal power and transmission length in the dispersion-managed $40-\mathrm{Gb} / \mathrm{s}$ nonlinear transmission. In this simulation, the use of NZDSF ( $D=4$ $\mathrm{ps} / \mathrm{nm} / \mathrm{km}$, loss $=0.23 \mathrm{~dB} / \mathrm{km}$ ) and Raman amplifier with $80-\mathrm{km}$ span is assumed.

of signal distortion, as a function of residual dispersion in the linear and nonlinear transmission cases. In Fig. 1, the solid line is the simulation result without considering the nonlinear effect and the dotted line is with consideration of the nonlinear effect. In this paper, we define the minimum $E O P$ as the EOP at the optimal dispersion compensation. In other words, the minimum EOP is the minimal EOP which can be obtained by using tunable dispersion compensation. And, note that the optimal dispersion-compensation ratio is different for each nonlinear transmission case.

Fig. 2 shows a contour graph of the minimum EOP at the optimal dispersion compensation as a function of signal power and transmission length in a dispersion-managed nonlinear transmission. It can be recognized that the minimum

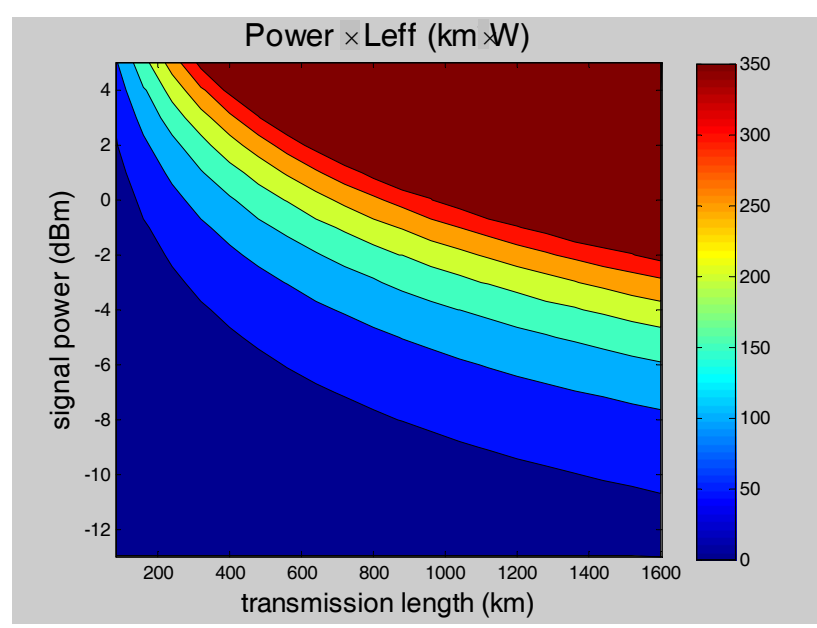

FIG. 3. A figure of merit for the efficiency of a nonlinear process, $\mathrm{I}_{0} \mathrm{~L}_{\mathrm{eff}}$, as a function of signal power and transmission length in the same condition as Fig. 2 . $\mathrm{I}_{0}$ is the optical intensity and $\mathrm{L}_{\text {eff }}$ is the effective length of the interaction region.

EOP increases when the signal power or transmission length increases. In the simulation, the use of NZDSF (non-zero dispersion-shifted fiber, $\mathrm{D}=4 \mathrm{ps} / \mathrm{nm} / \mathrm{km}$, loss $=0.23 \mathrm{~dB} / \mathrm{km}$ ) and Raman amplifier with $80-\mathrm{km}$ span is assumed. Because we need to conduct nonlinear simulations in many different cases of dispersion compensation to find the optimal dispersion compensation at each point, it takes lots of time to obtain the contour graph of the minimum EOP, such as Fig. 2. For example, the simulation results of Fig. 2 were obtained through more than 6000 nonlinear simulations.

In Fig. 3, we illustrate a contour graph of a figure of merit for the efficiency of a nonlinear process, $\mathrm{I}_{0} \mathrm{~L}_{\text {eff }}$, as a function of signal power and transmission length in the same condition as Fig. 2. Surprisingly, the contour graphs of Fig. 2 and Fig. 3 look similar, which makes us realize that there can be a strong relationship between the minimum $E O P$ and a figure of merit for the efficiency of a nonlinear process, $\mathrm{I}_{0} \mathrm{~L}_{\text {eff. }}$. From here in this paper, we will shorten the term, a figure of merit for the efficiency of a nonlinear process, to a figure of merit for a nonlinear process.

\section{FUNCTIONAL RELATION BETWEEN THE MINIMUM EOP AND A FIGURE OF MERIT FOR A NONLINEAR PROCESS}

We investigated the relation between the minimum EOP and a figure of merit for a nonlinear process, $\mathrm{I}_{0} \mathrm{~L}_{\text {eff. }}$ Fig. 4 shows the $x-y$ relation between the minimum EOP and a figure of merit for a nonlinear process by using all the simulation results of Fig. 2 and 3. From Fig. 4, we can say that the minimum EOP is a function of a figure of merit for a nonlinear process. In other words, although signal power or transmission length is changed, the minimum EOPs will be the same if a figure of merit for a nonlinear 


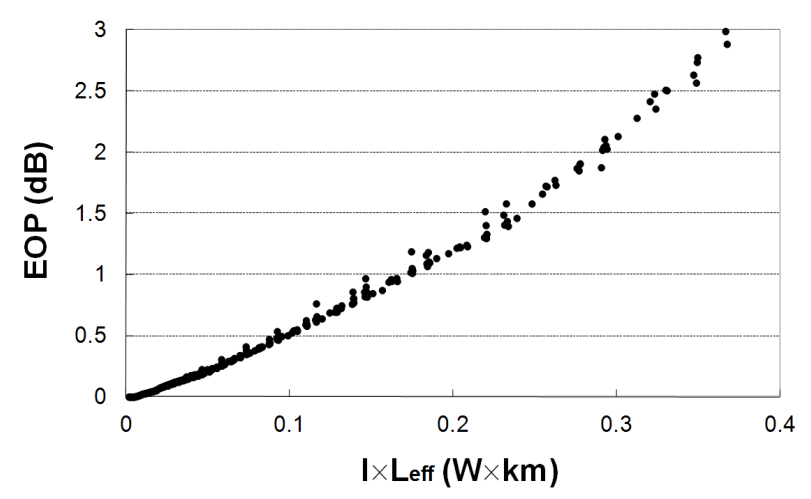

FIG. 4. The functional relation between the minimum EOP and a figure of merit for a nonlinear process, $\mathrm{I}_{0} \mathrm{~L}_{\mathrm{eff}}$, using all the simulation results of Fig. 2 and 3.

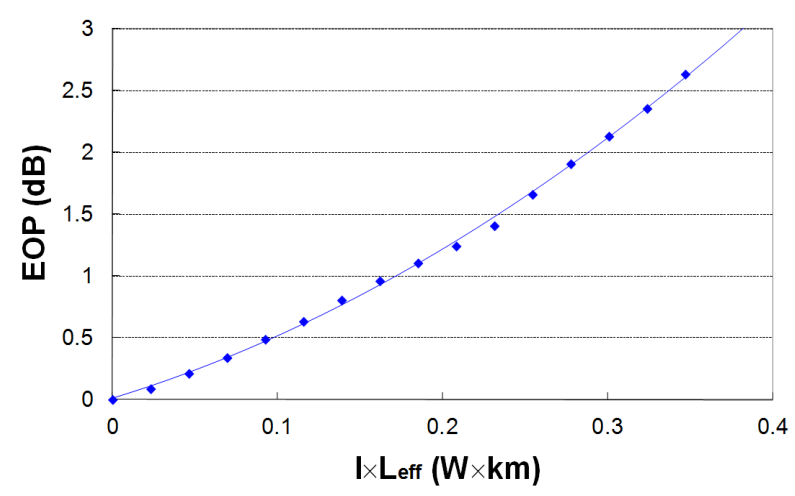

FIG. 5. The simulation result of only one case of a signal power, $-1 \mathrm{dBm}$, in the same condition as Fig. 2. The solid line is the $2^{\text {nd }}$ order polynomial fitting graph.

process, $\mathrm{I}_{0} \mathrm{~L}_{\mathrm{eff}}$, is the same. For example, the minimum EOP at $480-\mathrm{km}$ dispersion-managed transmission with a signal power of $0 \mathrm{dBm}(=1.05 \mathrm{~dB})$ is almost the same as the minimum EOP at $1520-\mathrm{km}$ dispersion-managed transmission with a signal power of $-5 \mathrm{dBm}(=1.01 \mathrm{~dB})$ because the values of $\mathrm{I}_{0} \mathrm{~L}_{\text {eff }}$ for these two cases are almost the same to a value of 0.175 . Some variation points in the functional relation in Fig. 4 seem to be caused by the discrete value of simulation parameters for dispersion compensation.

Therefore, we do not need to conduct nonlinear simulations in all the cases of signal power and transmission length to obtain the signal distortion in the dispersion-managed optical transmission. We need to conduct a simulation in only one case of a signal power, and then we can obtain the values of signal distortion in other cases using the functional relation shown in Fig. 4.

\section{USE OF THE FUNCTIONAL RELATION}

In this section, we will show an example of how to use the functional relation. Fig. 5 shows the simulation result in only one case of a signal power, - $1 \mathrm{dBm}$, in the same

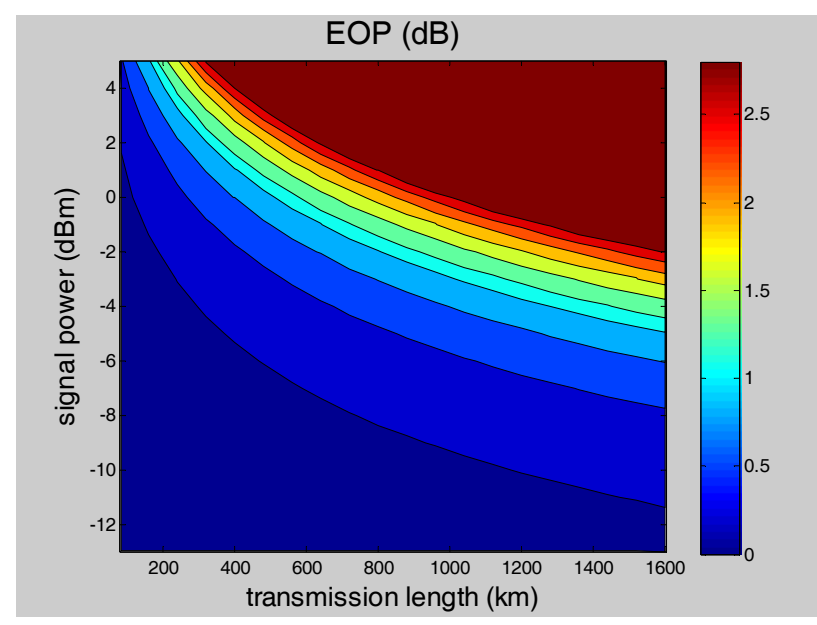

FIG. 6. Calculated EOPs using the functional relation between EOP and a figure of merit for a nonlinear process, $\mathrm{I}_{0} \mathrm{~L}_{\text {eff, }}$, in the same condition as Fig. 2.

condition of Fig. 2. Using the simulation results in Fig. 5, we can make a functional for $\mathrm{I}_{0} \mathrm{~L}_{\text {eff }}(\mathrm{x})$ and $\mathrm{EOP}(\mathrm{y})$. If we use the $2^{\text {nd }}$ order polynomial fit, we can obtain a fitting function, $\mathrm{y}=\mathrm{Ax}+\mathrm{Bx}+\mathrm{C}$, with the coefficients of $\mathrm{A}=$ 9.87, $\mathrm{B}=4.07$, and $\mathrm{C}=0.01$. The fitting function is illustrated as a solid line in Fig. 5.

Using the fitting function and the data of a figure of merit for a nonlinear process in Fig. 3, we can calculate EOPs in other cases of signal power and transmission length, and plot a contour graph of them, as shown in Fig. 6. The calculated EOPs in Fig. 6 are very similar to the EOPs obtained by a lot of nonlinear simulations in Fig. 2 .

Therefore, we can obtain the signal distortion in the dispersion-managed optical transmission system by conducting nonlinear simulations in only one case of a signal power. We do not need to conduct nonlinear simulations in all the cases of signal power and transmission length. Following the procedure of this section, anyone can use the proposed technique easily. In this example, the proposed technique reduces the number of nonlinear simulations to $5 \%$ of Fig. 2 .

\section{FUNCTIONAL RELATIONS IN OTHER SYSTEM CONDITIONS}

In this section, we investigate whether the functional relation between the minimum EOP and a figure of merit for a nonlinear process can be applied to other simulation conditions. Fig. 7 shows the relations between the minimum EOP and a figure of merit for a nonlinear process, $\mathrm{I}_{0} \mathrm{~L}_{\mathrm{eff}}$, in other simulation conditions. In the simulation of Fig. 7, we conducted several nonlinear simulations by changing one of system parameters: (1) the same condition as Fig. 4 (Raman amplifier with 80-km span, NZDSF (D = 4 $\mathrm{ps} / \mathrm{nm} / \mathrm{km}, 0.23 \mathrm{~dB}$ loss)), (2) 60-km amplifier span, (3) 100-km amplifier span, (4) $0.2 \mathrm{~dB}$ fiber loss, (5) $0.3 \mathrm{~dB}$ 


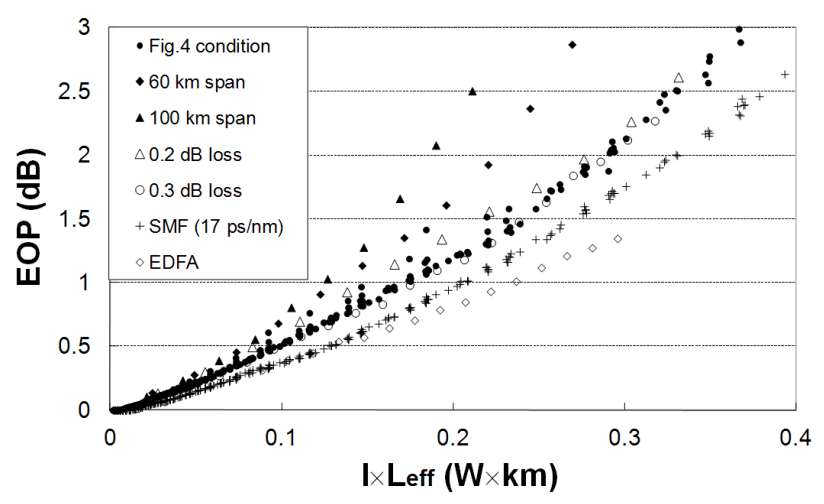

FIG. 7. The functional relations between the minimum EOP and a figure of merit for a nonlinear process, $\mathrm{I}_{0} \mathrm{~L}_{\mathrm{eff}}$, in other simulation conditions. (1) The same condition as Fig. 4 (Raman amplifier with $80-\mathrm{km}$ span, NZDSF (D = 4 $\mathrm{ps} / \mathrm{nm} / \mathrm{km}, 0.23 \mathrm{~dB}$ loss)), (2) 60-km amplifier span, (3) 100-km amplifier span, (4) $0.2 \mathrm{~dB}$ fiber loss, (5) $0.3 \mathrm{~dB}$ fiber loss, (6) SMF (D = $17 \mathrm{ps} / \mathrm{nm} / \mathrm{km})$, and (7) EDFA.

fiber loss, (6) SMF (single mode fiber, D = $17 \mathrm{ps} / \mathrm{nm} / \mathrm{km}$ ), and (7) using EDFA (erbium-doped fiber amplifier) instead of Raman amplifier.

From Fig. 7, we recognize that although the function between the minimum EOP and a figure of merit for a nonlinear process is changed depending on the system parameters, the functional relation itself still remains between the minimum EOP and a figure of merit for a nonlinear process. Therefore, in one nonlinear simulation condition, we can obtain the function for the minimum EOP and a figure of merit for a nonlinear process, and use it to reduce the nonlinear simulations. Even though we do not show all the simulation results we have conducted due to the lack of space, we confirmed the functional relation between the minimum EOP and a figure of merit for a nonlinear process in lots of different simulation conditions besides Fig. 7.

\section{ANALYTICAL DERIVATION FOR THE FUNCTIONAL RELATION}

Until this section, we have shown that the minimum EOP is a function of a figure of merit for a nonlinear process through our simulation results. In this section, we will derive it analytically for the case of a Gaussian pulse. From the earlier work, the analytic expression for the pulse broadening induced by dispersion is given by [12]

$$
\widetilde{U}(z, \omega)=\widetilde{U}(0, \omega) \exp \left[\frac{i}{2} \beta_{2} \omega^{2} z\right]
$$

where $\mathrm{U}(\mathrm{z}, T)$ is the normalized amplitude of the pulse, $\tilde{\mathrm{U}}(\mathrm{z}, \omega)$ is the Fourier transform of $\mathrm{U}(\mathrm{z}, T), \tilde{\mathrm{U}}(0, \omega)$ is the Fourier transform of the incident field at $\mathrm{z}=0$, and $\beta_{2}$ is the GVD (group-velocity dispersion) parameter. And, from the earlier work, the analytic expression for the pulse broadening induced by SPM is given by [13]

$$
U(z, T)=U(0, T) \exp \left\lfloor i \phi_{N L}(z, T)\right\rfloor
$$

where,

$$
\begin{aligned}
& \phi_{N L}(z, T)=|U(0, T)|^{2}\left(\gamma P_{0} L_{e f f}\right)=|U(0, T)|^{2} \phi_{\max } \\
& \phi_{\max }=\gamma P_{0} L_{e f f} ; \gamma=\frac{n_{2} \omega_{0}}{c A_{e f f}}
\end{aligned}
$$

where $\mathrm{P}_{0}$ is the optical peak power, $\mathrm{L}_{\text {eff }}$ is the effective length, $\omega_{0}$ is the carrier frequency, and $A_{\text {eff }}$ is the effective mode area. Therefore, the analytic expression for the pulse broadening when dispersion and SPM coexist can be written as [14]

$$
U(z, T)=F^{-1}\left\{\exp \left[\frac{i}{2} \beta_{2} \omega^{2} z\right] \cdot F\left\{U(0, T) \exp \left[i \phi_{N L}(z, T)\right]\right\}\right\}
$$

Consider the case of a Gaussian pulse for which the incident field is of the form

$$
U(0, T)=\frac{1}{\sigma_{0} \sqrt{2 \pi}} \exp \left[-\frac{T^{2}}{2 \sigma_{0}^{2}}\right]
$$

where $\sigma_{0}$ is the half-width of the pulse. When this Gaussian pulse, $\mathrm{U}(0, T)$, is incident at the input end of a fiber of length $\mathrm{L}$, the broadening factor can be solved by Eq. (5) and is given by [13]

$$
\frac{\sigma}{\sigma_{0}}=\left[1+\sqrt{2} \phi_{\max } D+\left(1+\frac{4}{3 \sqrt{3}} \phi_{\max }^{2}\right) D^{2}\right]^{1 / 2}
$$

where

$$
D=\frac{z}{L_{D}}=\frac{\beta_{2} z}{\sigma_{0}^{2}}
$$

$D$ represents the normalized distance for dispersion, or it can be considered as a measure of accumulated dispersion. $\mathrm{L}_{\mathrm{D}}$ is the dispersion length.

Now, consider the case of dispersion compensation. By using Eq. (7), the broadening factor of a Gaussian pulse with dispersion compensation can be written as

$$
E O P \equiv \frac{\sigma}{\sigma_{0}}=\left[1+\sqrt{2} \phi_{\max } D_{r e s}+\left(1+\frac{4}{3 \sqrt{3}} \phi_{\max }^{2}\right) D_{\text {res }}{ }^{2}\right]^{1 / 2}
$$

where $\mathrm{D}_{\text {res }}$ represents the residual dispersion after dispersion 
compensation. Because the broadening factor is also a measure of signal distortion and is proportional to EOP, we express that "EOP $\equiv$ broadening factor" in Eq. (9). To find the optimum dispersion compensation, we can take the partial derivative of the pulse broadening of Eq. (9) with respect to $D_{\text {res }}$ and obtain the following equation:

$$
\frac{\partial[E O P]}{\partial D_{r e s}}=\frac{1}{2} \cdot \frac{\left(\sqrt{2} \phi_{\max }+2 \cdot\left(1+\frac{4}{3 \sqrt{3}} \phi_{\max }^{2}\right) D_{r e s}\right)}{\left[1+\sqrt{2} \phi_{\max } D_{r e s}+\left(1+\frac{4}{3 \sqrt{3}} \phi_{\max }^{2}\right) D_{r e s}^{2}\right]^{1 / 2}}=0
$$

From Eq. (10), we can obtain the value of optimum dispersion compensation:

$$
D_{o p t}=\frac{-\sqrt{2} \phi_{\max }}{2+\frac{8}{3 \sqrt{3}} \phi_{\max }^{2}}=f\left(\phi_{\max }\right)
$$

From Eq. (11), we can recognize that the optimum value of dispersion compensation, $\mathrm{D}_{\text {opt }}$, is only a function of $\varphi_{\max }$. Now we substitute $D_{\text {opt }}$ back into Eq. (9) to obtain the signal distortion at the optimum dispersion compensation:

$$
\begin{aligned}
E O P_{\text {opt }} \equiv\left[\frac{\sigma}{\sigma_{0}}\right]_{o p t} & =\left[1+\sqrt{2} \phi_{\max } D_{\text {opt }}+\left(1+\frac{4}{3 \sqrt{3}} \phi_{\max }^{2}\right) D_{\text {opt }}^{2}\right]^{1 / 2} \\
& =\left[1+\sqrt{2} \phi_{\max } f\left(\phi_{\max }\right)+\left(1+\frac{4}{3 \sqrt{3}} \phi_{\max }^{2}\right)\left(f\left(\phi_{\max }\right)\right)^{2}\right]^{1 / 2} \\
& =g\left(\phi_{\max }\right) \\
& =h\left(I_{0} L_{e f f}\right)
\end{aligned}
$$

where

$$
\phi_{\max }=\gamma P_{0} L_{\text {eff }}=\frac{n_{2} \omega_{0}}{c A_{\text {eff }}} P_{0} L_{\text {eff }}=\frac{n_{2} \omega_{0}}{c} I_{0} L_{\text {eff }}
$$

Therefore, from Eq. (12), we can say that the pulse broadening, i.e. signal distortion, at the optimum dispersion compensation is a function of a figure of merit for a nonlinear process, $\mathrm{I}_{0} \mathrm{~L}_{\text {eff. }}$.

\section{THE RELATION IN THE CASE OF NON-OPTIMUM DISPERSION COMPENSATION}

Until this section, we have shown that there exists a functional relation between the minimum EOP and a figure of merit for a nonlinear process when the dispersion is optimally compensated. However, in this section, we investigate the relation between the minimum EOP and a figure of merit for a nonlinear process when the dispersion is not optimally compensated. Fig. 8 shows the relations between the minimum EOP and a figure of merit for a nonlinear process, $\mathrm{I}_{0} \mathrm{~L}_{\mathrm{eff}}$, in the case of non-optimum dispersion compensation. In Fig. 8, we show the results in the cases of $99 \%$ and

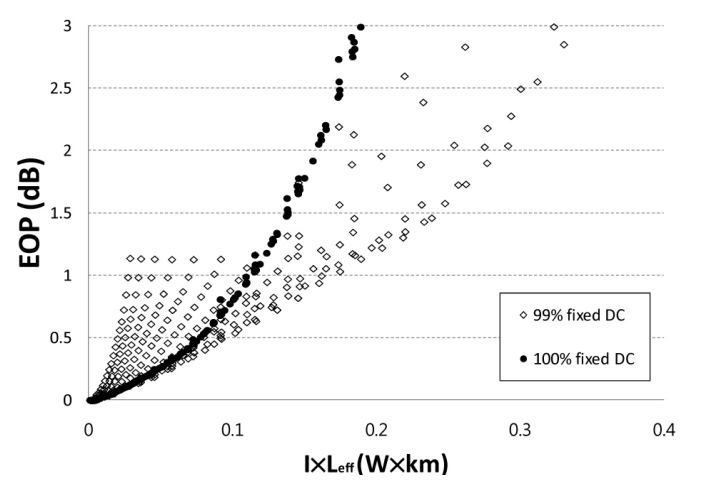

FIG. 8. The relations between the minimum EOP and a figure of merit for a nonlinear process, $\mathrm{I}_{0} \mathrm{~L}_{\mathrm{eff}}$, when the dispersion is not optimally compensated. The solid circles are the cases of $100 \%$ dispersion compensation. The hollow diamonds are the cases of $99 \%$ dispersion compensation.

$100 \%$ dispersion compensation. The system condition of Fig. 8 is identical to Fig. 2 and Fig. 4.

From the result of the $99 \%$ dispersion compensation case, we can recognize that the functional relation between the minimum EOP and a figure of merit for a nonlinear process does not remain when the dispersion is not optimally compensated. However, when the dispersion is compensated by $100 \%$, the functional relation still remains. We think that these results are somewhat obvious because only a nonlinear effect exists after $100 \%$ dispersion compensation. Thus the minimum EOP is also a function of a figure of merit for a nonlinear process in the case of $100 \%$ dispersion compensation, but not in other cases. Therefore, we can conclude that the functional relation only remains in the cases of the optimum and $100 \%$ dispersion compensation. In other words, we can say that the functional relation only remains in the cases when the nonlinear effect is dominant.

\section{CONCLUSION}

From our nonlinear simulation results for several cases, we showed that the minimum EOP obtained by tunable dispersion compensation is a function of a figure of merit for a nonlinear process, $\mathrm{I}_{0} \mathrm{~L}_{\text {eff, }}$, where $\mathrm{I}_{0}$ is the optical intensity and $L_{\text {eff }}$ is the effective length of the interaction region. We also showed an analytic derivation of the functional relation for the case of a Gaussian pulse.

Using this rule, we do not need to conduct nonlinear simulations in all the cases of signal power and transmission length to obtain the signal distortion in dispersion-managed optical transmission. Instead, we only need to conduct a simulation for one case of signal power and find the functional relation between the minimum EOP and a figure of merit for a nonlinear process, and then we can obtain the signal distortions in other cases using the discovered functional relation. This technique can reduce the number of nonlinear simulations to less than $10 \%$. This technique will reduce 
the time of nonlinear simulations in investigating, optimizing, or designing the nonlinear optical transmission systems.

\section{ACKNOWLEDGMENT}

This research was supported by Kyungsung University Research Grants in 2012.

\section{REFERENCES}

1. N. Kikuchi and S. Sasaki, "Analytical evaluation technique of self-phase modulation effect on the performance of cascaded optical amplifier systems," J. Lightwave Technol. 13, 868-878 (1995).

2. A. Cartaxo, "Impact of modulation frequency on crossphase modulation effect in intensity modulation-direct detection WDM systems," IEEE Photon. Technol. Lett. 10, 1268-1270 (1998).

3. R. S. Luís and A. V. T. Cartaxo, "Analytical characterization of SPM impact on XPM-induced degradation in dispersion -compensated WDM systems," J. Lightwave Technol. 23, 1503-1513 (2005).

4. A. V. T. Cartaxo, B. Wedding, and W. Idler, "Influence of fiber nonlinearity on the fiber transfer function: theoretical and experimental analysis," J. Lightwave Technol. 17, 18061813 (1999).

5. J. Wang and K. Petermann, "Small signal analysis for dis- persive optical fiber communication systems," J. Lightwave Technol. 10, 96-100 (1992).

6. S.-M. Kim and C.-H. Lee, "The efficient clock-extraction methods of NRZ signal for chromatic dispersion monitoring," IEEE Photon. Technol. Lett. 17, 1100-1102 (2005).

7. I. Shake, H. Takara, K. Uchiyama, and Y. Yamabayashi, "Quality monitoring of optical signals influenced by chromatic dispersion in a transmission fiber using averaged Q-factor evaluation," IEEE Photon. Technol. Lett. 13, 385-387 (2001).

8. S.-M. Kim and J.-Y. Park, "Chromatic dispersion monitoring of CSRZ signal for optimum compensation using extracted clock-frequency component," ETRI Journal 30, 461-468 (2008).

9. S.-M. Kim, "PMD effect on the clock-based optimum dispersion compensation monitoring technique," J. Opt. Soc. Korea 13, 112-115 (2009).

10. G. P. Agrawal, Nonlinear Fiber Optics, 4th ed. (Academic Press, San Diego, USA, 2007), section 1.3.3.

11. I. Kaminow and T. Li, Optical Fiber Telecommunications IV $B$ (Academic Press, San Diego, USA, 2002), Chapter 13.

12. G. P. Agrawal, Nonlinear Fiber Optics, 4th ed. (Academic Press, San Diego, USA, 2007), Chapter 3.

13. Govind P. Agrawal, Nonlinear Fiber Optics, 4th ed. (Academic Press, San Diego, USA, 2007), Chapter 4.

14. M. J. Potasek, G. P. Agrawal, and S. C. Pinault, "Analytic and numerical study of pulse broadening in nonlinear dispersive optical fibers," J. Opt. Soc. Am. B 3, 205-211 (1986). 